\title{
Optical Spectroscopy of Conductive Junctions in Plasmonic Cavities
}

\author{
O. Pérez-González, ${ }^{\dagger,}$ N. Zabala, ${ }^{\dagger,}{ }^{\mp}$ A. G. Borisov, ${ }^{\S}$ N. J. Halas, ${ }^{\prime}$ P. Nordlander, ${ }^{*}{ }^{\perp}$ and \\ J. Aizpurua*,†,§
}

${ }^{\dagger}$ Donostia International Physics Center (DIPC) and Centro de Física de Materiales, Centro Mixto CSIC-UPV/EHU, Paseo Manuel Lardizabal 4, 20018 Donostia-San Sebastián, Spain, ${ }^{\ddagger}$ Elektrizitatea eta Elektronika Saila, Euskal Herriko Unibertsitatea UPV-EHU 644 P.K., 48080 Bilbao, Spain, ${ }^{\S}$ Laboratoire des Collisions Atomiques et Moléculaires, UMR CNRS-Université Paris-Sud 8625, Bat. 351, 91405 Orsay CEDEX, France, and "Department of Electrical and Computer Engineering and Chemistry, Laboratory for Nanophotonics and ${ }^{\perp}$ Department of Physics, MS61, Laboratory for Nanophotonics, Rice University, Houston Texas 77005

\begin{abstract}
The optical properties of a nanoparticle dimer bridged by a conductive junction depend strongly on the junction conductivity. As the conductivity increases, the bonding dimer plasmon blueshifts and broadens. For large conductance, a low energy charge transfer plasmon also appears in the spectra with a line width that decreases with increasing conductance. A simple physical model for the understanding of the spectral feature is presented. Our finding of a strong influence of junction conductivity on the optical spectrum suggests that plasmonic cavities might serve as probes of molecular conductance at elevated frequencies not accessible through electrical measurements.
\end{abstract}

KEYWORDS Plasmonics, nanoparticle, dimer

$\square$ lectronic transport through molecules is a vibrant field in nanoscience, promising advanced technological applications in nanoelectronics. During the past decade, many fundamental advances in our understanding of molecular transport at DC or low AC frequencies have been achieved. ${ }^{1-6}$ In molecular electronics, it is clearly of significant importance to understand transport at gigahertz or higher frequencies. While there has been significant theoretical effort devoted to understanding electron transport through molecular devices and quantum dots at elevated frequencies, ${ }^{7-9}$ standard electrical transport measurements cannot be performed in this regime due to the strong capacitive coupling between electrodes.

Also during the past decade, there have been significant developments in the understanding of the optical properties of plasmonic nanostructures. ${ }^{10,11} \mathrm{~A}$ unique feature of plasmonic nanostructures is the sensitive dependence of their plasmon resonance energies on nanostructure geometry and dielectric environment. ${ }^{12-14}$ When two nanoparticles are placed next to each other, forming a nanoparticle dimer configuration, the plasmon modes of the individual nanoparticles interact, resulting in hybridized dimer plasmon modes with energies that can be strongly red shifted relative to the plasmon modes of the individual nanoparticles. ${ }^{15-17}$ In particular, the dipolar bonding dimer plasmons (BDP) with large localized charge distributions of opposite sign on either

* To whom correspondence should be addressed. E-mail: (P.N.) nordland@rice.edu; (J.A.) aizpurua@ehu.es.

Received for review: 05/15/2010

Published on Web: 07/12/2010 side of the junction can give rise to enormously enhanced local electromagnetic fields in the junction. ${ }^{18,19}$

In several recent experiments and theoretical studies it has been shown that when a conductive overlap between two closely spaced nanoparticles is established, the optical spectrum and local field distribution can be strongly modified. ${ }^{20-28}$ When a conduction path between two nanoparticles opens, a charge transfer plasmon (CTP), involving both a polarization of the charge distribution of the individual nanoparticles and an oscillating electrical current across the junction, emerges. The energy of the CTP depends very sensitively on the junction profile and thus indirectly on the conductance of the junction between the two particles. ${ }^{21}$ In spite of these studies, no clear picture has emerged of the CTP and how its properties depend on the shape and conductive properties of the junction.

In this paper, we present a theoretical investigation of how the optical properties of a plasmonic conductive cavity (PCC) consisting of a strongly coupled (nontouching) nanoparticle dimer are influenced by the presence of a conductive bridge across the junction between the nanoparticles. This system can be viewed as a simple model of two nanoparticles linked by an ensemble of conductive molecules. We show that the optical properties of the PCC depend strongly on the conductance of the bridge. This finding suggests that molecular conductance at elevated frequencies can be probed by optical means in this geometry and offers a possible strategy for measuring such conductances in the visible and infrared region of the spectrum.

The geometry of our simple model system is depicted in Figure $1 \mathrm{a}$. The permittivities $\varepsilon(\omega)$ of the materials, $\mathrm{Au}^{29}$ and 


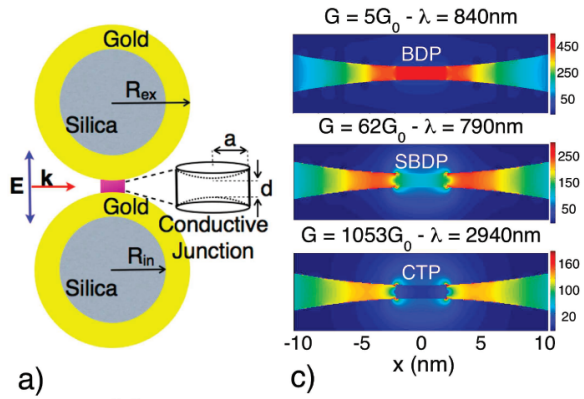

a)

c)

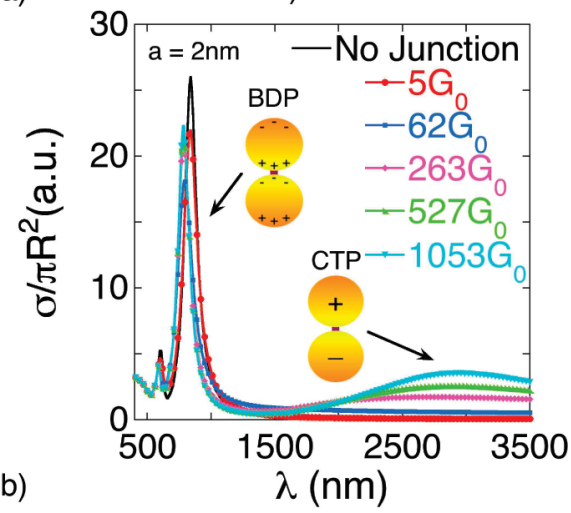

FIGURE 1. (a) Schematic representation of the PCC. A nanoshell dimer connected by a cylindrical conductor of radius $a$ and internal length $d$. The radius of the silica core is $R_{\text {int }}=45 \mathrm{~nm}$, the outer gold surface $R_{\text {ext }}=55 \mathrm{~nm}$. k is the wavevector of the incoming electromagnetic wave. (b) Extinction spectra of a PCC for $a=2 \mathrm{~nm}$ as a function of junction conductance. Insets illustrate the charge distributions for the dipolar BDP and the CTP. (c) Near-field maps at the resonance wavelengths for some of the systems in (b): BDP at $\lambda=840 \mathrm{~nm}$ (top); SBDP at $\lambda=790 \mathrm{~nm}$ (middle); CTP with at $\lambda=$ $2940 \mathrm{~nm}$ (bottom).

silica, ${ }^{30}$ are taken from the literature. The dimer is assumed to be suspended in vacuum. For simplicity, to illustrate that conductance matters the conductive bridge is modeled as a pure conductor with conductivity $\kappa_{\mathrm{J}}$, that is

$$
\varepsilon(\omega)=1+i \frac{4 \pi \kappa_{J}}{\omega}
$$

The conductance $G$ of the junction can be calculated analytically, $G=\hat{G}\left(R_{\text {ext }}, a, d\right) \kappa_{\mathrm{J}}$, where $\hat{G}$ depends on the geometrical parameters $R_{\text {ext }}, a$, and $d$. In the limit of a thin junction $a \ll$ $d, R_{\text {ext }}$, the conductance can be approximated by $G=\kappa_{\mathrm{J}} \pi a^{2} /$ $d$. For the interparticle distance considered here $(d=1 \mathrm{~nm})$, the conductance scales as $a^{2}$ up to $a \sim 3 \mathrm{~nm}$, then depends approximately linearly on $a$. To connect with the terminology for molecular conductance, we will vary $\kappa_{\mathrm{J}}$ so that the total conductance of the junction is equal to an integer number $n$ of quantum units of conductance, that is, $G=n G_{0}$ where $G_{0}=2 e^{2} / h \approx 77.5 \mu \mathrm{S}$. The optical response and the induced local fields of the system are calculated by solving Maxwell's equations exactly using the boundary element method (BEM). ${ }^{31}$

In Figure 1b, we show the extinction spectra of the PCC as a function of junction conductance over a large wave- length scale. The peak around $840 \mathrm{~nm}$ is the hybridized dipolar BDP resulting from the capacitive coupling of the individual nanoshell plasmon modes. ${ }^{15}$ As the conductance of the junction increases, the energy of the BDP blueshifts and its width changes. For the largest conductance, a new plasmon mode appears around $\lambda \approx 3000$ $\mathrm{nm}$. This is the CTP where in addition to a polarization of the charge distribution of each nanoshell, an electric current flows across the junction and the net charge of the individual nanoshells will oscillate in time. The CTP is particularly prominent in the spectrum when the junction conductivity becomes similar to the conductivity of the metal particles.

In Figure 1c, we show the local electric field distributions in the junction for the BDP and CTP. For low conductance $\left(G=5 G_{0}\right)$, a field is still present across the junction, allowing for a significant capacitive coupling between the two nanoparticles. As the conductivity increases, the local field is progressively expelled from the molecular junction, reducing the capacitive coupling across the cavity. For intermediate values of conductance $\left(G=62 G_{0}\right)$, it can clearly be seen that the local field across the conductive bridge has disappeared. In this case, the capacitive coupling between the two nanoshells is reduced and results in a slight blueshift of the BDP. We will refer to this blue-shifted BDP as a Screened BDP (SBDP). In the bottom panel of Figure 1c, we show the field distribution for the CTP for a large conductance. The contour plot clearly shows that the junction fields outside the conductive bridge are also strongly reduced. In this limit, the conductive junction acts as a short circuit, drastically reducing the potential difference between the two nanoshells. We address now in more detail the spectral features and tendencies of both the BDP and the CTP as optical fingerprints of the conductance of the junction.

In Figure $2 \mathrm{a}-\mathrm{c}$, we show the extinction spectra in the short wavelength region of the spectrum, near the BDP, for three different junction radii and several different conductances. For small values of the conductance, the energy of the $\mathrm{BDP}$ remains unaltered with a monotonic increase in line width with increasing conductance (see Figure 2e,f). For intermediate conductance, the BDP blueshifts and the SBDP is formed. As the conductance becomes larger, the energy of the SBDP remains unchanged but its line width decreases. This behavior is found systematically for the three different sizes of the conductive junctions in Figure $2 \mathrm{a}-\mathrm{c}$. Conductance thresholds for the formation and narrowing of the SBDP clearly depend on the geometry of the junction. In Figure 2e,f, we show how the width and shift of the SBDP depend on junction conductivity. These results clearly show a correlation between the spectral behavior of the BDP and the junction conductivity.

The line width dependence on the junction conductance can be qualitatively understood from a simple resistor model (Figure 3a) where the PCC is modeled as a serial combination of resistors $r_{\mathrm{Au}}$ (gold particles) and $r_{\text {junction }}$ (molecular junc- 

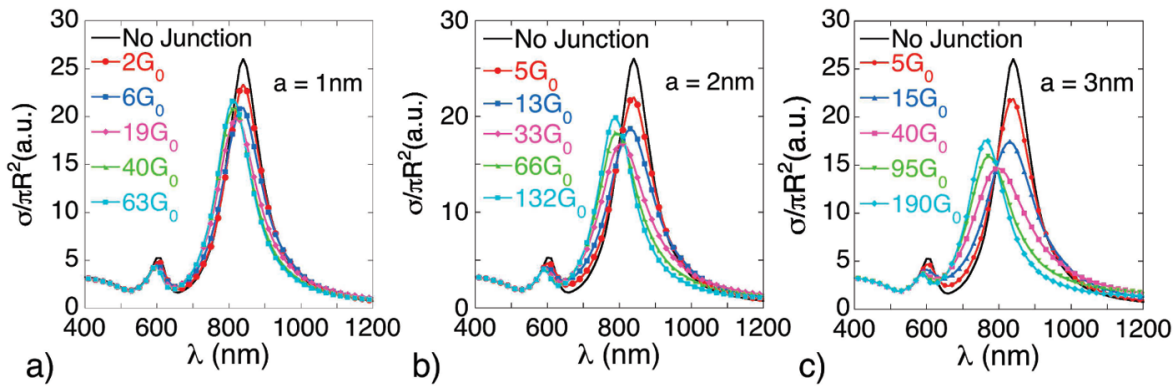

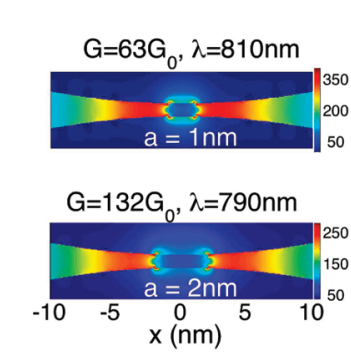

d)

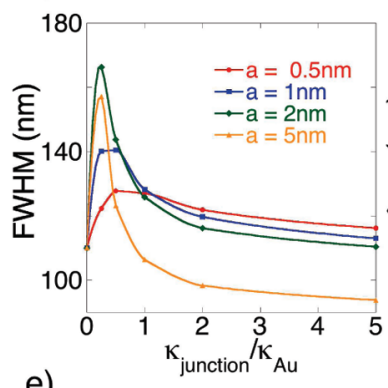

e)

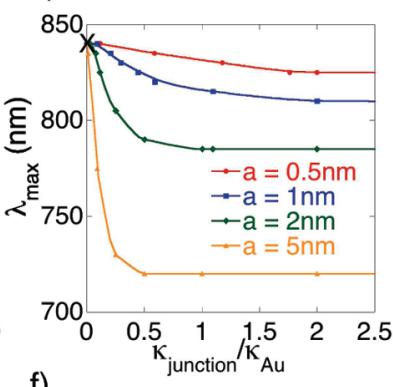

FIGURE 2. Extinction spectra of the PCC as a function of conductance for different radii: $a=1 \mathrm{~nm}$ (a), $a=2 \mathrm{~nm}$ (b), and $a=3 \mathrm{~nm}$ (c). (d) Near-field map at resonance wavelengths: $a=1 \mathrm{~nm}$ and $G=63 G_{0}$ (top); $a=2 \mathrm{~nm}$ and $G=132 G_{0}$ (bottom). (e) Full width at half-maximum (fwhm) of the BDP resonance as a function of conductivity $\kappa_{\mathrm{J}}=\kappa_{\text {junction }}$ of the molecular junction for different radii of the junction. The conductivity is normalized to the conductivity of gold. (f) Evolution of the BDP to a SBDP peak position for the same conditions as in (e).

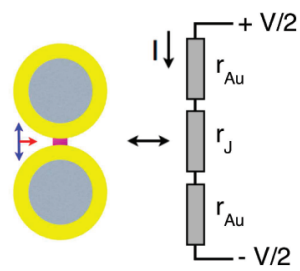

a)

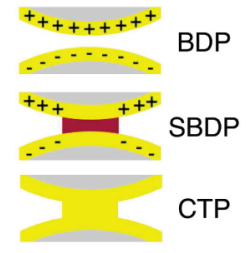

b)

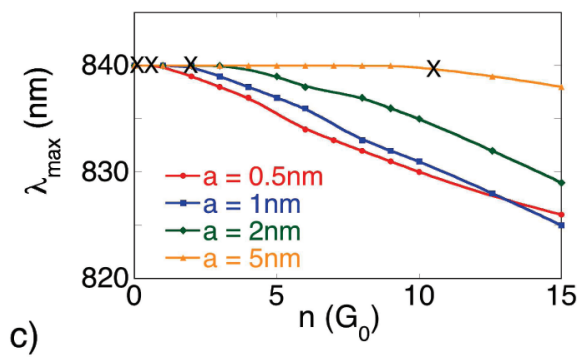

FIGURE 3. (a) Resistor model describing the response of the PCC. Each nanoshell is represented by a resistor $r_{\mathrm{Au}}$, and the conductive bridge by $r_{\mathrm{J}}$. The voltage from the incident field is $V$. (b) Schematics illustrating the reduction of the capacitive coupling across the junction when the junction conductance is increased for the BDP (top), SBDP (middle), and CTP (bottom). (c) Blueshift of the BDP as a function of junction conductance $G$ for different radii in the low conductance regime.

tion). The power dissipated in the junction $W_{\mathrm{J}}$, when a potential $V$ is applied, is

$$
W_{\mathrm{J}}=\frac{V^{2} r_{\mathrm{J}}}{\left(2 r_{\mathrm{Au}}+r_{\mathrm{J}}\right)^{2}}
$$

For low junction conductance $\left(r_{J} \gg r_{\mathrm{Au}}\right)$, the dissipation in the junction scales as $W_{\mathrm{J}} \approx 1 / r_{\mathrm{J}} \approx \kappa_{\mathrm{J}}$. In this low conduc- tance limit, the junction dissipation and hence the broadening of the SBDP increases linearly with the conductivity of the molecular junction. For large junction conductance $\left(r_{J}\right.$ $\left.\ll r_{\mathrm{Au}}\right)$, the dissipation in the junction scales as $W_{\mathrm{J}} \approx r_{\mathrm{J}} \approx 1 / \kappa_{\mathrm{J}}$, that is, a further increase of junction conductivity produces a decrease of the dissipation and hence, a reduced line width. This is exactly the situation observed in Figure $2 \mathrm{e}$.

The magnitude of the blueshift increases with junction radius $a$. In the absence of the junction, a large Coulomb attraction between the two nearby sides of nanoparticles is present for the BDP, as illustrated in the top panel of Figure 3b. For the SBDP, as illustrated in the middle panel, the Coulomb attraction is reduced since the surface charges facing the molecular junction have been shorted. The decrease in capacitive coupling with increasing $a$ can be inferred from the near-field plots in Figure $2 d$, which shows that a larger junction removes more surface charge and results in lower field enhancements than a small junction. For small junction radii, the blueshift is proportional to the cross sectional area of the junction $a^{2}$, in qualitative agreement with Figure 2f.

Both the SBDP and CTP plasmon involve oscillatory charge transport across the junction. To qualitatively understand the threshold conductance for the appearances of the SBDP and the CTP it is useful to relate the time involved in the electron transport processes $\tau_{\mathrm{SBDP}}$ and $\tau_{\text {СTP }}$ to the time of the corresponding optical cycles.

As illustrated in Figure 3b, the SBDP is formed when the surface charges on the opposite sides of the junction have had time to neutralize. To estimate the magnitude of the charge that needs to be transported in each cycle, we note 
that for the BDP, the local electric field enhancement in the junction is $E^{\text {loc }}=\left[\left(2 R_{\text {ext }}\right) / d\right] E^{\text {ext }}$, where $E^{\text {ext }}$ is the external electric field. ${ }^{32}$ This local electric field enhancement corresponds to a surface charge density of $\sigma_{\mathrm{BDP}}=E^{\mathrm{loc}} / 4 \pi$. For a small junction, one can assume the surface charge to be constant across the junction, resulting in a total charge $Q_{\text {BDP }}$ $=\pi a^{2}\left(E^{\mathrm{loc}} / 4 \pi\right)$ to be transported in each cycle. The current through the junction is $I=G E^{\text {loc }} d$. The time for this transport $\tau_{\mathrm{BDP}}=Q_{\mathrm{BDP}} / I=a^{2} / 4 G d$. For the SBDP to be formed, this time scale must be shorter than a fraction of an optical cycle. If we take this fraction to be one-fourth, for example, this time scale must be shorter than $t_{\mathrm{BDP}}=\pi / 2 \omega_{\mathrm{BDP}}$. We thus obtain the threshold conductance for the SBDP

$$
G_{\mathrm{SBDP}}=\frac{\omega_{\mathrm{BDP}}}{2 \pi} \frac{a^{2}}{d}
$$

As discussed in Figure 2, the conductance threshold for the SBDP depends on junction geometry. By factoring out the geometrical quantities in eq 3 , the threshold for the SBDP can be expressed in terms of conductivity as $\kappa_{\mathrm{J}}>\omega_{\mathrm{SBDP}} / 2 \pi^{2}$, that is, the condition for the appearance of the SBDP in absolute terms is related to the conductivity of the junction rather than its conductance. We can observe this effect in Figure 2f, where a common position of the threshold conductivity is marked with a cross for all molecular dimensions.

In Figure 3c, we show the initial shift of the BDP with increasing conductance in the low conductivity regime, for several junctions of different radii. The threshold conductances obtained from eq 3 are marked with crosses for each radius. A systematic trend can be observed for all cases: a range of low conductance where the BDP peak remains at the same spectral position $(\lambda=840 \mathrm{~nm})$, followed by a blueshift of the BDP, eventually reaching the SBDP. The onset of the blueshift occurs exactly for the threshold conductance eq 3 .

A similar argument can be applied to understand the conductance required to sustain the CTP. When the CTP is fully formed, the plasmonic charge will be concentrated on the outside hemispheres of the two nanoshells and the total field across the junction will be negligible (bottom panels in Figures $1 \mathrm{c}$ and $3 \mathrm{~b}$ ). The plasmon induced field in the junction is thus $E^{\text {loc }}=-E^{\text {ext }}$. The total charge that needs to be transported in each cycle is $Q_{\text {CTP }}=\left(R_{\text {ext }}^{2} / 4\right) E^{\text {ext }}$ and the amplitude of the instantaneous local field is $2 E^{\text {ext }}$. The time scale for this charge transport is $\tau_{\text {СTP }}=Q_{\mathrm{CTP}} / I=\left(R_{\text {ext }}^{2} t\right.$ $4 G d)\left(E^{\text {ext }} / 2 E^{\text {ext }}\right)$. As for the SBDP, the CTP requires that $\tau_{\text {СтP }}$ be a fraction of the optical cycle time $t_{\text {СТP }}=\pi / 2 \omega_{\text {СTP. }}$. This leads to the following expression for the conductance threshold

$$
G_{\mathrm{CTP}}=\frac{\omega_{\mathrm{CTP}}}{4 \pi} \frac{R_{\mathrm{ext}}^{2}}{d}
$$
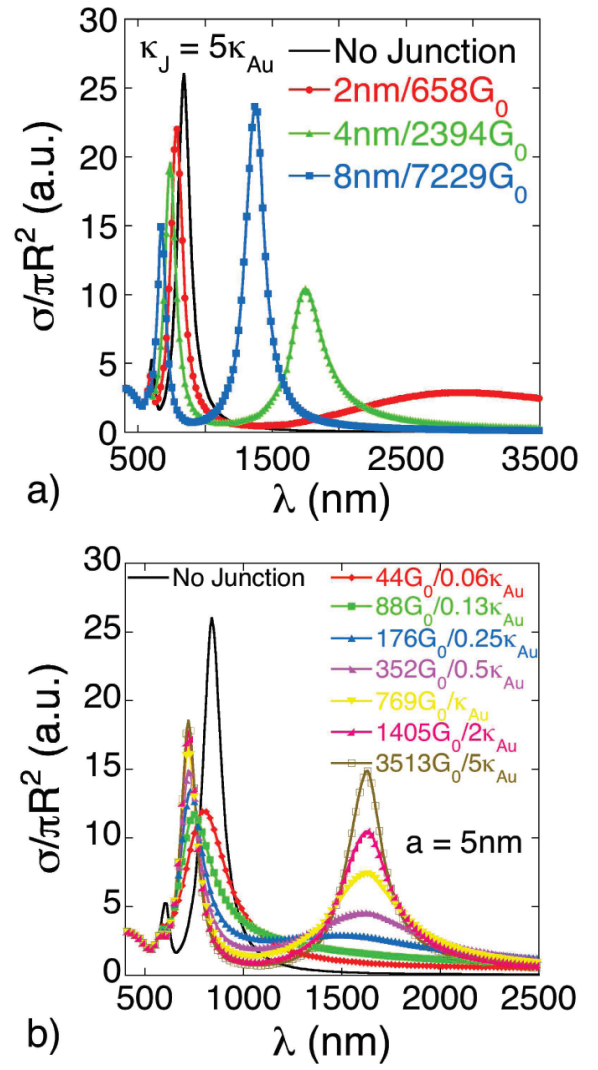

FIGURE 4. (a) Extinction spectra for dimers connected by junctions of varying radii $a$ and conductivity $\kappa_{\mathrm{J}}=5 \kappa_{\mathrm{Au}}$. (b) Extinction spectra for a junction of fixed radius $a=5 \mathrm{~nm}$ but varying conductance.

This expression shows that the CTP is related to the conductance of the junction rather than its conductivity and that the CTP requires significantly larger conductances than the SBDP.

In Figure 4, we explore situations where the conductivity of the junction is similar in magnitude to that of gold. First, we analyze the case of a junction with a conductivity 5 times larger than that of gold in Figure 4a. In this limit, the dimer acts as one continous nanostructure, rather than a coupled pair of nanoparticles. As for plasmonic nanoparticles in general, the plasmon frequencies depend strongly on geometry. This is clearly evident in the figure, which shows a strong redshift of the CTP with decreasing junction radius. The figure also shows a significant narrowing of the CTP for larger conductance due to the reduced dissipation for large conductance.

To more clearly illustrate the effect of dissipation, in Figure $4 \mathrm{~b}$ we show spectra for a PCC with a junction of fixed size. The figure shows that the energy of the CTP resonance remains fixed as expected in the large conductance limit. The significant narrowing of the CTP resonance with increasing conductance is due to the reduced dissipation and is in agreement with our predictions using the simple resistor model eq 2. For the junction in Figure $4 \mathrm{~b}$, our time scale analysis of the CTP, eq 4 , gives $G_{\text {СТP }}=400 G_{0}$. This result is in excellent agreement with the figure which shows that a CTP is clearly well developed above $352 G_{0}$. For the junction 
in Figure $1 \mathrm{~b}$, eq 4 predicts $G_{\text {CTP }}=225 G_{0}$ which is consistent with the fully developed CTP for $G=263 G_{0}$.

A blueshift of the BDP and the emergence of a CTP as a function of conductive overlap between two nanoparticles is an effect that has been discussed theoretically and observed experimentally both in nanoshell dimers and in cylindrical infrared gap antennas where metallic junctions bridge the gap. ${ }^{23,25}$ In these cases, the conductivity of the junctions are the same as the surrounding nanoparticles. In such a situation, the resulting plasmon modes are controlled only by the geometry of the junction. The results for the infrared antennas ${ }^{25}$ are in excellent agreement with the present results. As the diameter and consequently the conductance of the junction is increased, the BDP blueshifts and a low energy CTP appears on the red side of the BDP. Our simple estimate for the appearance of the CTP, eq 4, applies also for this cylindrical geometry and predicts a CTP for a junction radius of 10 $\mathrm{nm}$ in almost perfect agreement with the theoretical simulations. ${ }^{25}$

The present calculation assumed a very simple frequency independent conductivity of the bridge between the two nanoparticles. This assumption was made to show that the optical response of the PCC was sensitive to the conductance of the junction. In a realistic situation, one cannot assume a frequency independent conductance. For instance, if the junction consists of stacked molecules, one would expect that a tunneling or ballistic electron could induce both vibrational and low energy electronic excitations of the molecules. Such processes are likely to induce sharp resonances in the frequency dependent conductance. For metallic nanowire junctions, one would expect that density of states effects and electron-electron interactions could also introduce a strong frequency dependence of the conductance.

To experimentally probe situations where the junction conductance is expected to exhibit sharp resonances, one would need to tune the energy of the PCC plasmons across the conductance peak and also in between such resonances. The plasmon resonances of the PCC resonances can of course be tuned across the visible region of the spectrum and into the far-infrared region by simply changing the geometry of the structure. However, a more convenient approach for tuning would be embedding the structure in a dielectric medium. Our calculation shows that both the SBDP and CTP resonances are indeed highly tunable in this manner, and that both resonances readily can be shifted by much larger energies than the energies of typical molecular vibrations (around $100 \mathrm{meV}$ ).

To take full advantage of the SBDP and CTP as fingerprints of electron transport processes at optical frequencies, it is possible to optimize the PCC by using smaller diameter nanoparticles, plasmonic materials of lower conductivities, or plasmonic dimers with more red-shifted plasmon modes. Such systems will present smaller threshold conductivities, and could thus be used to detect lower conductance values.
In summary, we have studied the optical behavior of a metallic dimer connected by conductive junctions and have identified two different regimes of its optical response. In the low conductance regime, the optical response depends on the conductivity rather than the conductance of the junction. The optical spectrum exhibits a bonding dimer mode BDP which blueshifts into a screened bonding dimer plasmon SBDP as the conductivity increases. In the large conductance regime, a charge transfer plasmon CTP appears prominently on the red side of the SBDP. This mode is characterized by an oscillating electric current flowing across the junction. Using simple universal time scale arguments, we have established conductivity and conductance thresholds for the appearance of these features. We believe that the study of spectral changes in plasmonic nanocavities linked by conductive bridges such as molecules, nanotubes, and nanowires can be used to probe electrical transport at visible frequencies, a regime not accessible through electrical measurements.

Acknowledgment. This project was supported by the Robert A. Welch Foundation, Grants C-1220 and C-1222(P.N., N.J.H.), Etortek project inanoGUNE from the Basque Government (BG), Project FIS2007-066711-C02-00 from the Spanish MICINN, Grant IT-366-07 from BG-UPV/EHU and Project EUI2008-03816 CUBiHOLE from the internationalization national plan of the Spanish MICINN within the EU ERA-NET projects (J.A., N.Z., O.P.G.). Computational resources were provided by IZO-SGIker (UPV/EHU, MICINN, BG, ESF).

\section{REFERENCES AND NOTES}

(1) Park, H. K.; Park, J. W.; Lim, A. K. L.; Anderson, E. H.; Alivisatos, A. P.; McEuen, P. L. Nature 2000, 407, 57-60.

(2) Nazin, G. V.; Qiu, X. H.; Ho, W. Science 2003, 302, 77-81

(3) Dadosh, T.; Gordin, Y.; Krahne, R.; Khivrich, I.; Mahalu, D.; Frydman, V.; Sperling, J.; Yacoby, A.; Bar-Joseph, I. Nature 2005, 436, 677-680.

(4) Venkataraman, L.; Klare, J. E.; Tam, I. W.; Nuckolls, C.; Hybertsen, M. S.; Steigerwald, M. L. Nano Lett. 2006, 6, 458-462.

(5) Banerjee, P.; Conklin, D.; Nanayakkara, S.; Park, T. H.; Therien, M. J.; Bonell, D. A. ACS Nano 2010, 4, 1019-1025.

(6) Liao, J.; Agustsson, J. S.; Wu, S.; Schonenberger, C.; Calame, M.; Leroux, Y.; Mayor, M.; Jeannin, O.; Ran, Y. F.; Liu, S. X.; Decurtins, S. Nano Lett. 2010, 10, 759-764.

(7) Hyldgaard, P. Phys. Rev. B 2008, 78, 165109

(8) Wu, B. H.; Cao, J. C. Phys. Rev. B 2010, 81, No. 085327

(9) Heyl, M.; Kehrein, S. Phys. Rev. B 2010, 81, 144301.

(10) Pelton, M.; Aizpurua, J.; Bryant, G. W. Laser Photon. Rev. 2008, 2, 136-159.

(11) Lal, S.; Link, S.; Halas, N. J. Nat. Photonics 2007, 1, 641-648.

(12) Bryant, G. W.; de Abajo, F. J. G.; Aizpurua, J. Nano Lett. 2008, 8, $631-636$.

(13) Li, Z. P.; Shegai, T.; Haran, G.; Xu, H. X. ACS Nano 2009, 3, 637 642 .

(14) Wang, W.; Li, Z. P.; Gu, B.; Zhang, Z. Y.; Xu, H. X. ACS Nano 2009, 3, 3493-3496.

(15) Nordlander, P.; Oubre, C.; Prodan, E.; Li, K.; Stockman, M. I. Nano Lett. 2004, 4, 899-903.

(16) Kim, D. S.; Heo, J.; Ahn, S. H.; Han, S. W.; Yun, W. S.; Kim, Z. H. Nano Lett. 2009, 9, 3619-3625.

(17) Yang, S. C.; Kobori, H.; He, C. L.; Lin, M. H.; Chen, H. Y.; Li, C.; Kanehara, M.; Teranishi, T.; Gwo, S. Nano Lett 2010, 10, 632637 
(18) Ward, D. R.; Halas, N. J.; Ciszek, J. W.; Tour, J. M.; Wu, Y. P.; Nordlander, P.; Natelson, D. Nano Lett. 2008, 8, 919-924.

(19) Zhang, Z.; Weber-Bargioni, A.; Wu, S. W.; Dhuey, S.; Cabrini, S.; Schuck, P. J. Nano Lett. 2009, 9, 4505-4509.

(20) Atay, T.; Song, J. H.; Nurmikko, A. V. Nano Lett. 2004, 4, 1627 1631.

(21) Romero, I.; Aizpurua, J.; Bryant, G. W.; de Abajo, F. J. G. Opt. Express 2006, 14, 9988-9999.

(22) Danckwerts, M.; Novotny, L. Phys. Rev. Lett. 2007, 98, No. 026104.

(23) Lassiter, J. B.; Aizpurua, J.; Hernandez, L. I.; Brandl, D. W. Romero, I.; Lal, S.; Hafner, J. H.; Nordlander, P.; Halas, N. Nano Lett. 2008, 8, 1212-1218.

(24) Zuloaga, J.; Prodan, E.; Nordlander, P. Nano Lett. 2009, 9, 887 891
(25) Schnell, M.; Garcia-Etxarri, A.; Huber, A. J.; Crozier, K.; Aizpurua, J.; Hillenbrand, R. Nature Photon. 2009, 3, 287-291.

(26) Large, N.; Abb, M.; Aizpurua, J.; Muskens, O. L. Nano Lett. 2010, 10, $1741-1746$.

(27) van der Molen, S. J.; Liao, J.; Kudernac, T.; Anderson, J. S.; Bernard, L.; Calame, M.; van Wees, B. J.; Feringa, B. L.; Schonenberger, C. Nano Lett. 2009, 9, 76-80.

(28) Chau, Y. F.; Lin, Y. J.; Tsai, D. P. Opt. Express 2010, 18, 35103518

(29) Johnson, P. B.; Christy, R. W. Phys. Rev. B 1972, 6, 4370-4379.

(30) Palik, E. D. Handbook of Optical Constants of Solids; Academic: San Diego, CA, 1985.

(31) de Abajo, F. J. G.; Howie, A. Phys. Rev. B 2002, 65, 115418.

(32) Le, F.; Brandl, D. W.; Urzhumov, Y. A.; Wang, H.; Kundu, J.; Halas, N. J.; Aizpurua, J.; Nordlander, P. ACS Nano 2008, 2, 707-718. 